\title{
An Analysis of Loss-Free Data Aggregation for High Data Reliability in Wireless Sensor Networks
}

\author{
Stephen Brown \\ Dept. of Computer Science \\ Maynooth University \\ Email: stephen.brown@nuim.ie
}

\begin{abstract}
Data aggregation is am important feature in Wireless Sensor Networks, used primarily to reduce energy use. This paper extends our previous results, which showed that data aggregation can improve the reliability of data delivery rather than degrading it as previously assumed. These previous results were based on the use of scaleable aggregation functions, such as SUM, COUNT, MIN, MAX which work independently of the network size. In this paper we extend these results to consider the reliability and energy efficiency of lossless data delivery with the semi-scaleable aggregation function APPEND, and determine the boundary conditions under which the data reliability can be maintained without an increase in the energy cost. These new results show that lossless aggregation using the APPEND aggregation function can provide improved reliability with reduced energy usage in certain conditions.
\end{abstract}

Index Terms-Wireless Sensor Networks, Data Aggregation, Aggregation Functions, Reliability.

\section{INTRODUCTION}

In-network data aggregation is used in Wireless Sensor Networks (WSNs) to reduce the volume of data traffic: this is a key issue in WSNs where both energy and bandwidth are constrained [1] [2] [3] [4]. The aggregation is done by executing aggregation functions at aggregation points on the path from the sensing nodes to the gateway [5]. Aggregation typically results in a reduced data set: either by removing redundancy (for example similar readings from nearby nodes), or by producing a summary of the data (e.g. min and max values) [6]. More advanced techniques may use more sophisticated techniques to minimise the data loss during aggregation: for example compression [7], outlier detection [8], or network coding [9]. Many sensor network protocols support aggregation, for example: TAG, Directed Diffusion, PEGASIS, DBMAC, LEACH, Cougar; see [5] for a comprehensive overview and comparison of these.

As shown in a previous study by the author, aggregation can be used to increase the reliability of data delivery from the sensing nodes to the gateway [10]. This results from using the energy saved by the traffic reduction to increase the retransmit limit (and thus the reliability) of the packets sent containing aggregated data. As shown through analysis and simulation, the reliability can be increased with no increase in energy

This publication has emanated from research supported in part by a research grant from Science Foundation Ireland (SFI) and is co-funded under the European Regional Development Fund under Grant Number 13/RC/2077 (compared to the non-aggregated case). And in most cases, both an decrease in energy and an increase in reliability can be achieved.

Data distribution and collection tasks can be performed optimally on tree networks [11]. In [12] the minimum Steiner tree is compared with the shortest path tree, and found to be optimal. Other approaches use clustering (e.g. LEACH [13]), multi-path forwarding (e.g. Synopsis Diffusion [14]), or hybrid schemes [15]. Much recent work focuses on security issues in data aggregation [16] [17] [18]. Retransmissions can be used to improve reliability: as shown for PSFQ in [19], hop-by-hop error recovery is significantly more effective than end-to-end recovery in a wireless environment.

Recent work on data aggregation functions in Wireless Sensor Networks addressed the use of different approaches to aggregation. These include Karnaugh Arrays [20], Set Similarity Functions [21], and Fitting Functions [22]. These all address different approaches to optimising data size reduction during aggregation. In many cases all the data is required to be delivered, rather than a summary of the data (as provided by most aggregation functions such as MIN, MAX, etc.). The results presented here address this situation, and evaluate the fundamental implications and limitations of not reducing data size during aggregation.

The standard equation (Eqn. 1) for determining the optimal packet size [23], provides the motivation for considering increasing the packet size during aggregation in order to increase the efficiency of the protocol (i.e. the completeness of the data collected for a given amount of energy expended).

$l_{\text {opt }}=\frac{-h \ln \left(1-p_{b}\right)-s q r t\left[-4 h \ln \left(1-P_{b}\right)+h^{2} \ln \left(1-P_{b}\right)^{2}\right]}{2 \ln \left(1-P_{b}\right)}$

In this paper we extend our previous results, which applied to lossy [5] or scaleable aggregation functions, to consider non-lossy or non-scaleable aggregation functions. These functions are non-scaleable in that they are limited in the numbr of nodes that can be supported. In this analysis, we consider aggregation functions where the limit is provided by the maximum packet size (and the per-node data element size). This extension allows us to consider the case where the aggregation function merely extends a list of data to be sent to the gateway, and explore the boundaries of the reqgion 

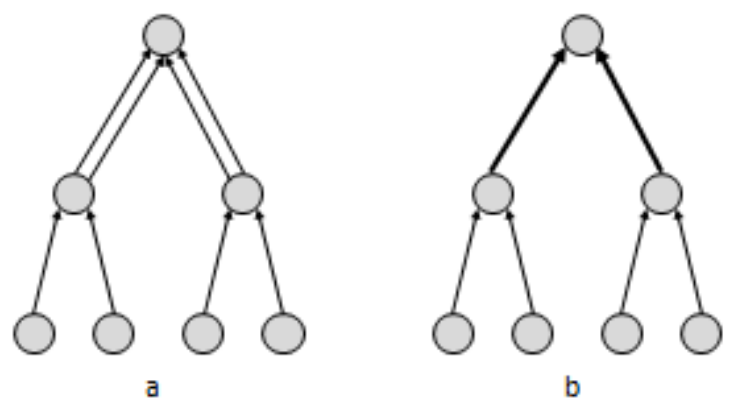

Fig. 1. Example Aggregation Tree: (a) non-aggregated, (b) aggregated

where the increased packet loss rate (determined by the Bit Error Rate or BER) due to the increased packet length in more than compensated for by the reduced packet count (due to aggregation). Our results show that, in some cases with limited network size, this form of aggregation can outperform an unaggregated approach, and even provide higher delivery reliability.

\section{Methodology}

We model data transfer from all the nodes to a root node (assumed to be a gateway), over a spanning tree, by evaluting the probabilities of data reception with retransmissions. As a first-order approximation, all acknowledgements are assumed to be successful, and take no time. The analysis does not include the generation and maintenance of the spanning tree, or the mechanism for triggering the data transfers (which could be, for example, timer-driven or polled). Each packet is acknowledged locally, and retransmitted (up to the retransmit limit) if the acknowledgement times out. A sample configuration is shown in figure 1 including a 2 -way spanning tree of depth 2 hops. In (a) the data is not aggregated, and each packet sent from a leaf node is then re-sent by the intermediate node to the root node. In (b) the data is aggregated, represented by the increased arrow width, and the data sent by the leaf nodes is aggregated in the intermediate nodes, and the aggregated data is sent in a single packet to the root. We consider the case where the aggregation function is APPEND, and all data is sent in a single packet: therefore the depth of the network is limited by the maximum packet size in this analysis.

The aggregated packets require more re-transmissions to maintain the required delivery reliability, given the increased Packet Error Rate (PER) due to the increased packet size. However, the important factor, is that this increase is less than the overall reduction in the number of packets sent. This is shown in Table I. As the degree of aggregation increases, represented by the value of $\mathrm{N}$, the number of retransmissions (t) required to maintain the reliability (here set at 99.9\%) increases at a lower rate. The efficiency of this is shown in the ' $t / N$ ' column: the number of transmissions required per byte decreases as the packet length increases. For example, with 16 data bytes the PER has more than doubled, but the
TABLE I

Transmissions REQUIRED VS DATA SiZE $(\mathrm{BER}=0.00008)$

\begin{tabular}{|l|r|r|r|r|r|}
\hline $\mathrm{N}$ & Bits & PER(1 xmit) & PER(t xmits) & $\mathrm{t}$ & $\mathrm{t} / \mathrm{n}$ \\
\hline 1 & 120 & 0.010 & 0.001 & 1.49 & 1.49 \\
\hline 2 & 128 & 0.010 & 0.001 & 1.51 & 0.75 \\
\hline 4 & 144 & 0.012 & 0.001 & 1.55 & 0.39 \\
\hline 8 & 176 & 0.014 & 0.001 & 1.62 & 0.2 \\
\hline 16 & 240 & 0.019 & 0.001 & 1.75 & 0.11 \\
\hline 32 & 368 & 0.029 & 0.001 & 1.95 & 0.06 \\
\hline 64 & 624 & 0.049 & 0.001 & 2.29 & 0.04 \\
\hline 127 & 1128 & 0.0826 & 0.001 & 2.82 & 0.02 \\
\hline
\end{tabular}

number of transmissions required (on average) to maintain the reliability above $99.9 \%$ has only increased by a factor of 1.17. The Bit Error Rate (BER) of 0.00008 (or 80 ppm) is selected to provide a reliability of $99 \%$ for a 1-byte (data) packet. Retransmission are then used to provide the required reliability of $99.9 \%$ - on average this takes 1.5 retransmissions for the 1-byte packet. The value of $t$ is calculated to provide the required reliability. Note that a protocol overhead of 14 bytes is used, representative of the PHY and MAC overhead for 802.15.4 [24], and this is included in the Bits calculation. The equations used are shown in section II-A.

Table II shows the calculations for a much higher bit error rate (BER) of 0.006 (or 6000ppm), providing a PER of $51.4 \%$ for a single packet transmission. This represents a weak wireless link, where the SNR is significantly decreased due to either distance or noise, and less than half of the packets are successfully transferred. Note that this shows several significant issues:

- The infeasibility, in general, of providing high reliability under these conditions

- Note the very large number (on average) of transmissions required for a 127-byte packet. This ensures that all 127 bytes are transferred witha reliability of $99.9 \%$. To ensure that 127 individual packets were transferred with an overall reliability of $99.9 \%$ would require that each packet was transferred with a reliability of $99.9992 \%$ which would require 16.93 transmissions on average, resulting in a total of $127 * 16.93=2150$ transmissions. This shows that at very high error rates, and with relatively large packet sizes, aggregation is more effective at maintaining high reliabilities. The tradeoff point for 127 data bytes is a BER of 0.00495 which corresponds to a singletransmission PER of $44.87 \%$. If the link reliability is lower than $55 \%$ then it is more effective to use either lossy aggregation or transmit each packet individually (for a single hop). In practice, the transmit counts are so large as to render this level of reliability essentially unachievable with this level of bit errors.

\section{A. Variables and Equations}

- T: total nodes in the wireless sensor network

- D: data bytes in a packet

- H: packet overhead size in bytes (MAC overhead plus a 1-byte discriminator) 
TABLE II

Transmissions REQUIRED VS DATA SIZE (BER=0.006)

\begin{tabular}{|l|r|r|r|r|r|}
\hline $\mathrm{N}$ & Bits & PER(1 xmit) & PER(t xmits) & $\mathrm{t}$ & $\mathrm{t} / \mathrm{n}$ \\
\hline 1 & 120 & 0.514 & 0.001 & 1.49 & 10.39 \\
\hline 2 & 128 & 0.537 & 0.001 & 11.11 & 5.56 \\
\hline 4 & 144 & 0.579 & 0.001 & 12.67 & 3.17 \\
\hline 8 & 176 & 0.653 & 0.001 & 16.22 & 2.03 \\
\hline 16 & 240 & 0.764 & 0.001 & 25.67 & 1.60 \\
\hline 32 & 368 & 0.891 & 0.001 & 59.74 & 1.87 \\
\hline 64 & 624 & 0.977 & 0.001 & 291 & 4.56 \\
\hline 127 & 1128 & 0.999 & 0.001 & 6127 & 48.24 \\
\hline
\end{tabular}

- $\mathrm{N}$ : total packet size in bytes $(=\mathrm{D}+\mathrm{H})$

- $\mathrm{C}_{i}$ : the number of children node $i$ has

- $\mathrm{PER}_{i}$ : Packet Error Rate, for packets received by node $i$

- BER: Bit Error Rate

- $\mathrm{ATX}_{i}$ : Average number of transmits required by node $i$ for its parent to successfully receive the packet, using retransmissions

- $\operatorname{Pr}(\mathrm{rx})$ : probability of receiving a packet of length $\mathrm{N}$ bytes with a transmit limit of $\mathrm{Tx}_{\text {lim }}$

- $\mathrm{Tx}_{\text {lim }}$ : transmit limit (i.e. $\mathrm{Tx}_{l i m}-1$ retransmissions)

- ARF: the aggregation re-transmission factor: this is an aggregation parameter

The standard equations for PER, ATX are:

$$
\begin{gathered}
P E R=1-\left((1-B E R)^{N}\right) \\
A T X=1 /(1-P E R) \\
N=H+D
\end{gathered}
$$

Note that this equation for ATX is a first-order estimate, as it assumes infinite retransmissions.

The probability that a node receives a packet, with retransmissions, is given by:

$$
\operatorname{Pr}(r x)=1-\left(P E R^{T x_{l i m}}\right)
$$

The probability that node $i$ receives data from all $\mathrm{C}_{i}$ children is given by:

$$
\begin{aligned}
\operatorname{Pr}\left(r x_{i}, a l l\right) & =\prod_{i=1}^{C_{i}}\left(1-\left(P E R^{T x_{l i m}}\right)\right) \\
& =\left(1-\left(P E R^{T x_{l i m}}\right)\right)^{C_{i}}
\end{aligned}
$$

As shown in [10], packets containing multiple, aggregated data values require an increased retransmission limit in order to provide high reliability (i.e. high percentage of data delivery). The value (value ${ }_{i}$ ) of a packet sent by node $i$ is measured by the count of aggregated data values contained. Thus a nonaggregated packet has a value 1 , and a packet containing 64 aggregated values has a value 64 . Using this, and the ARF, the transmit limit $\left(\mathrm{Tx}_{\text {lim }}(i)\right.$ for aggregated packets transmitted by node $i$ is calculated as follows:

$$
T x_{\text {lim,agg }}(i)=A R F * \text { value }_{i} * T x_{\text {lim }}
$$

\section{B. Metrics}

The different scanarios are compared using the total energy required for $99 \%$ success (i.e. that $99 \%$ of the data arrives at the root node). The parameters ( $\mathrm{Tx}_{l i m}$ and ARF) are calculated to provide the closest value to $99 \%$ in each case.

- Energy cost: as a first-order estimate, this is calculated as being proprtional to the number of packets sent (as this represents the length of time that a transmitter must be active and the corresponding received must be active, given an ideal scheduling algorithm).

$$
\text { Energy } \propto \sum_{i=1}^{i=T} A T X_{i}
$$

\section{Aggregation functions}

1) Expanded Scaleable Functions: In-network aggregation functions such as COUNT, SUM, MIN, and MAX [25] are scaleable in that the size of the data does not increase as the number of data sources increases. The functions MEAN and STANDARD-DEVIATION, frequently regarded as In-server aggregation functions [25], can be added to the range of scaleable functions, by including auxiliary variables required to perform incremental evaluation of the function [26]. This requires a slight increase in the fixed data length, over the size of the actual data values themselves, but this is a fixed overhead, and does not significantly decrease the efficiency of the aggregation (as shown in II-D below).

The probability that the root receives all the data (Success) is calculated by the product of the probabilities that each child successful sends it (aggregated) data to its parent (assuming node 1 is the root node):

$$
\operatorname{Pr}(\text { Success })=\prod_{i=2}^{T}\left(1-\left(P E R^{T x_{l i m}(i)}\right)\right)
$$

where $\operatorname{Tx}_{\text {lim }}(i)$ is calculated for each node using Eqn 7.

2) Semi-Scaleable Functions: The APPEND aggregation function results in the data size growing in direct proportion to the number of data entries: new data is appended to the existing list. This is not fully scaleable in two ways: firstly, once the packet size is exceeded, different algorithms may be required to handle multi-packet data. And secondly, from a reliability viewpoint, the number of required retransmissions grows in proportion to the number of data entries. This factor limits the reliability, but in section II-D below the limits of the applicability of this function are explored, and it is shown that in certain circumstances the APPEND function can be used without decreasing reliability (or increasing the costs of reliability) compared to the non-aggregated case. We are treating this as a semi-scaleable function, by limiting the number of nodes in the analysis, so that the data can fit within a single packet. This is not an unreasonable approach for many wireless sensor networks. It is future work to consider the impact when multiple packets are required.

The probability that the root receives all the data (Success) is calculated by the product of the probabilities that each child 
successful sends it data to the root node via all the intervening parents (assuming node 1 is the root node, and that node $i$ is at a depth of $d_{i}$ ):

$$
\operatorname{Pr}(\text { Success })=\prod_{i=2}^{T}\left(1-\left(P E R^{T x_{l i m}}\right)\right)^{d_{i}}
$$

Note that $\mathrm{Tx}_{l i m}$ is constant in this case.

\section{Aggregation model}

The aggregation model is based on a spanning tree, where each leaf reports its raw data to its parent. The parent then applies its aggregation function (if defined), and reports it's aggregated data to its parent in turn. This is continued until the root node (gateway) receives all the aggregated data. The algorithms for scheduling and retransmissions are not included in the model: they are assumed to be optimal, providing a bestcase analysis.

\section{E. Scenarios considered}

Four different scenarios are considered, each containing approximately 64 nodes, as shown in Fig. 2 (where w is the fan-out at each node, $\mathrm{d}$ is the depth in hops, and $\mathrm{n}$ is the total number of nodes):

- NARROW(a): A deep tree, of depth 63 hops, and each parent having 1 child (giving 64 nodes).

- MEDIUM-2(b): A medium-breadth tree, of depth 6 hops, and each parent having 2 children (giving 63 nodes).

- MEDIUM-4(c): A medium-breadth tree, of depth 3 hops, and each parent having 4 children (giving 81 nodes).

- BROAD(d): A broad tree, of depth 2 hops, and each parent having 8 children (giving 73 nodes).

In each case the energy required to provide $99 \%$ successful delivery of data to the root node is calculated. For the nonaggregagated cases, the number of transmission required at each hop for every data packet is calculated to provide the required overall probability of a packet reaching the root node. For the aggregated cases, the number of tranmissions required at each hop for the aggregated data is calculated, such that the probability of the eachh data byte in the final aggregated packet delivered to the root is equal to the required reliability. In both cases, this ensures that each byte of data is received with the required reliability.

\section{RESULTS}

\section{A. Numerical Results}

In all cases the BER used is 0.0001 , providing for a PER of $1 \%$ (and a data reliability of $99 \%$ ) for a packet with 1 data byte. The maximum number of transmits is set to an integer value that enables the non-aggregated case to meet the required reliability of delivery of all the data to the root node (of 99\%). The maximum transmissions is increased by a factor consisting of the number of data elements*the policy factor for aggregated data (to reflect the increased value of the data) as shown in [10]. In each case, the data delivered by the sensing nodes is 1 Byte long, and a packet overhead of

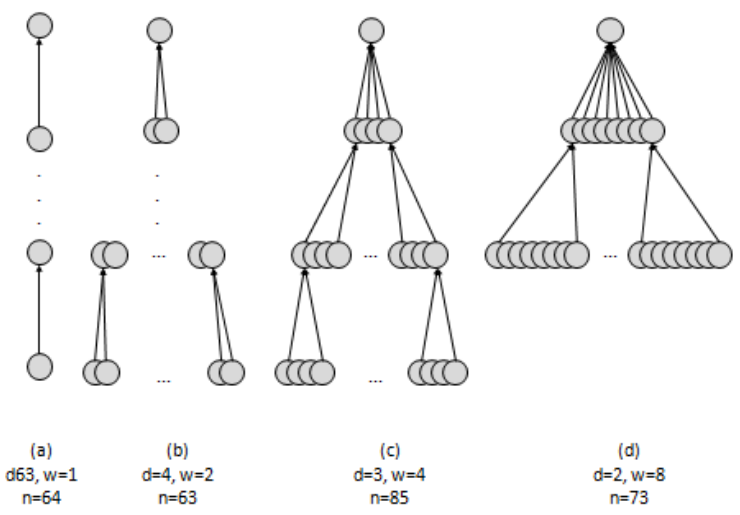

Fig. 2. Configurations Evaluated

TABLE III NARROW TREE

\begin{tabular}{|l|r|r|r|r|}
\hline $\begin{array}{l}\text { Aggregation } \\
\text { Aggregation }\end{array}$ & $\begin{array}{r}\text { Reliability } \\
\text { Reliability }\end{array}$ & $\begin{array}{r}\text { Max } \\
\text { Transmits }\end{array}$ & $\begin{array}{r}\text { Policy } \\
\text { Factor }\end{array}$ & $\begin{array}{r}\text { Avg. Total } \\
\text { Transmits Needed }\end{array}$ \\
\hline None & $99.65 \%$ & 3 & N/A & 2040 \\
\hline APPEND & $99.44 \%$ & 3 & 2 & 65 \\
\hline
\end{tabular}

14-Bytes is used (to represent the non-payload contents of a typical 802.15.4 PDU).

For the NARROW scenario (see Table III), the results show the large number of packets required for every data packet to be delivered separately up the tree in the non-aggregated case. The APPEND aggregation results in a significantly lower energy usage for the same level of reliability.

For the MEDIUM-2 scenario (see Table IV), a lower re-transmit limit (Max Transmits) is required, as the nonaggregaged packets only require 4 hops rather than 63 to reach the root. The results show the ratio of packets required for the non-aggregated case is still significantly larger. As for the NARROW scenario, the APPEND aggregation results in a significantly lower energy usage for the same level of reliability.

For the MEDIUM-4 scenario(see Table V), a lower re-

TABLE IV

MEDIUM-2 TREE

\begin{tabular}{|l|r|r|r|r|}
\hline $\begin{array}{l}\text { Aggregation } \\
\text { Aggregation }\end{array}$ & $\begin{array}{r}\text { Reliability } \\
\text { Reliability }\end{array}$ & $\begin{array}{r}\text { Max } \\
\text { Transmits }\end{array}$ & $\begin{array}{r}\text { Policy } \\
\text { Factor }\end{array}$ & $\begin{array}{r}\text { Avg. Total } \\
\text { Transmits Needed }\end{array}$ \\
\hline None & $99.86 \%$ & 5 & N/A & 650 \\
\hline APPEND & $99.44 \%$ & 5 & 2 & 127 \\
\hline
\end{tabular}

TABLE V

MEDIUM-4 TREE

\begin{tabular}{|l|r|r|r|r|}
\hline $\begin{array}{l}\text { Aggregation } \\
\text { Aggregation }\end{array}$ & $\begin{array}{r}\text { Reliability } \\
\text { Reliability }\end{array}$ & $\begin{array}{r}\text { Max } \\
\text { Transmits }\end{array}$ & $\begin{array}{r}\text { Policy } \\
\text { Factor }\end{array}$ & $\begin{array}{r}\text { Avg. Total } \\
\text { Transmits Needed }\end{array}$ \\
\hline None & $98.43 \%$ & 3 & N/A & 231 \\
\hline APPEND & $99.99 \%$ & 3 & 1 & 84 \\
\hline
\end{tabular}


TABLE VI

BROAD TREE

\begin{tabular}{|l|r|r|r|r|}
\hline $\begin{array}{l}\text { Aggregation } \\
\text { Aggregation }\end{array}$ & $\begin{array}{r}\text { Reliability } \\
\text { Reliability }\end{array}$ & $\begin{array}{r}\text { Max } \\
\text { Transmits }\end{array}$ & $\begin{array}{r}\text { Policy } \\
\text { Factor }\end{array}$ & $\begin{array}{r}\text { Avg. Total } \\
\text { Transmits Needed }\end{array}$ \\
\hline None & $99.81 \%$ & 3 & N/A & 138 \\
\hline APPEND & $98.88 \%$ & 3 & 1 & 72 \\
\hline
\end{tabular}

transmit limit (Max Transmits) is again required, as the nonaggregaged packets require fewer hops to reach the root. The results show the ratio of packets required for the nonaggregated case is still significantly larger. As for the previous scenarios, the APPEND aggregation results in a significantly lower energy usage for the same level of reliability.

For the BROAD scenario(see Table VI), the same retransmit limit (Max Transmits) is required, due to selecting an integer value. The results show the ratio of packets required for the non-aggregated case is twice the level requiored for the aggregated case. As for the previous scenarios, the APPEND aggregation results in a significantly lower energy usage for the same level of reliability.

In all cases, the size of the network is limited by the size of the data (here, 1 Byte has been used) and the maximum number of nodes on any branch of the tree (from 63 for the NARROW scenario, to 16 in the MEDIUM-4 scenario) and the maximum PDU size.

\section{B. Initial Real-World Results}

These initial results were gained using Atmel STK600Atmega128RFA1 devices, and a transmit power of $-16.5 \mathrm{dBm}$ (the receiver sensitity is specified to be $-100 \mathrm{dBm}$ at $250 \mathrm{kbps}$ ). Three cases were selected to send 100 bytes of data upstream representing data aggregated and sent from a child node to its parent in the tree: one 100-byte packet, ten 10-byte packets, and one 100-byte packet. Application-level acknowledgements and unlimited re-transmits were used to deliver the data. IEEE 802.15.4 channel 18 was used (to minimise interference from Wi-Fi APs on IEEE802.11 channels 1 and 13).

In each case, multiple experiments were run-note that the varying number of retransmits caused each experiment to take different lengths of time (as shown on the x-axis in Fig. 3). The different signal-strength conditions were achieved by moving the receiver until it reported the required behaviour. A channel analyser was used to determine the background noise levels: on this channel they were mainly below $-100 \mathrm{dBm}$, with very occasional spikes at $-90 \mathrm{dBm}$.

The resulting patterns of transmission are shown in Fig. 3. For each experiment, the number of transmissions required to deliver 100 bytes is shown. The Strong Signal results show the expected case where one hundred 1-byte packets, ten 10byte packerts, and one 100-byte packet are usually needed. The Varying Signal results show a situation (experiments 916) where over six hundred 1-byte packets were needed to deliver the data.

Table VII shows a summary of the results: the Avg. Tx Time is an indication of the relative energy required in each
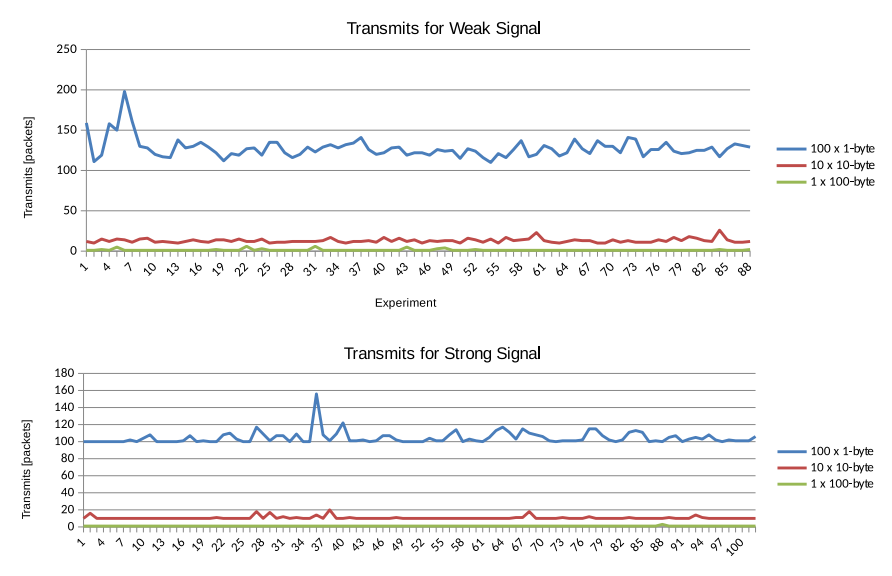

Experiment

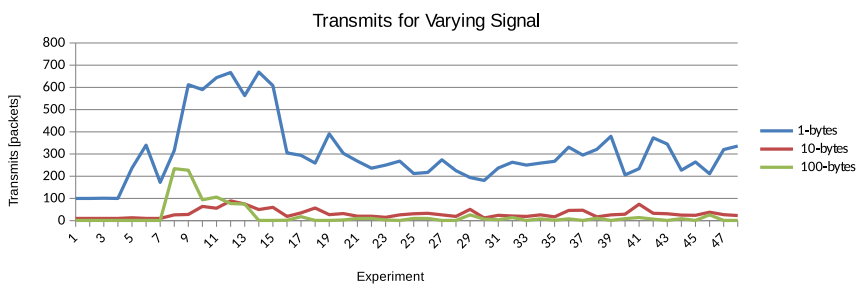

Fig. 3. Transmission Patterns

TABLE VII

RESULT SUMMARY

\begin{tabular}{|l|r|r|r|r|r|r|}
\hline & \multicolumn{4}{|c|}{ Avg. Tx Count [pkts] } & \multicolumn{4}{|c|}{ Avg. Tx Time [ms] } \\
\hline Packet Size [bytes] & 1 & 10 & 100 & 1 & 10 & 100 \\
\hline Good & 105.0 & 10.6 & 1.0 & 50.4 & 8.2 & 3.7 \\
\hline Weak & 127.6 & 13.0 & 1.3 & 61.3 & 10.0 & 4.9 \\
\hline Varying & 308.6 & 31.1 & 21.8 & 148.1 & 23.8 & 79.4 \\
\hline
\end{tabular}

case (the best-case time required on air to transmit 100 bytes of data). In general, the 100-byte packet size produces the greatest effeciency (for 100\% data delivery): but note that the Varying configuration shows a case where 100 bytes is above the optimum packet size. Compare to the patterns shown in figure 3 , it can be seen that the devices were effectively out of range, and approximnately 200 re-transmissions were required to deliver the 100-byte packet. This motivates the need for realtime monitoring of the packet error rate (PER) in determining the maximum packet length to use for semi-scaleable functions (such as APPEND) during data aggregation.

\section{CONCLUSION}

These results show that under certain conditions reliable delivery of aggregated data can be achieved with a wider range of aggregation functions than previous considered. In paticular, the APPEND function, which aggregates without data loss or data size reduction, can perform well compared to the non-aggregated case. In applications where all data must be delivered, to a high level of reliability, in sizeconstrained networks (where all the data from a particular branch of the tree can fit in a single packet), this provides 
significant improvements in energy efficiency. Alternatively, the energy savings from the improved efficiency can be used to provide a higher level of reliability. Or these two factors can be balanced, providing improved reliability with reduced energy usage. By monitoring the packet error rate (PER), and applying Eqn. 1, a protocol can determine dynamically when the APPEND function can be effectively utilized to provide enhanced efficiency.

The reader should note that these constraints may limit the application of the APPEND aggregation function in the general case, but many sensor networks are of limited size, with limited data being delivered, and in these situations this aggregation function can be used to significantly improve the reliability of the network while also increasing its lifetime by reducing the energy requirement. It is possible that a hybrid aggregation function might be used, that delivers loss-free extended data, perhaps including geo-location and additional environmental readings, for critical values (such as the MIN and MAX), while summarising the non-critical values (using MIN, MAX, MEAN, and other lossy aggregation functions).

A future extension is to consider the fundamental impact of allowing multiple packets to be used for the aggregated data, removing the restrictions on the network size and depth. Also, the impact of security concerns, which is a recent focus for aggregation in wireless sensor networks, has not been addressed in this analysis, and it will be interesting to include these into the analysis and see whether the benefits still exist in these cases. Additional future work will address a more detailed energy model considering the impact of lost acknowledgements, simulations to evaluate a broader range of scenarios and gather a broader set of data relating to the need for large numbers of retransmissions, and the implementation of real-world experiments using our existing, 256node testbed. These experiments will also explore how well existing routing algorithms (to generate the spanning tree), and scheduling algorithms (to control the reporting timing of the nodes) work with the APPEND aggregation function.

\section{ACKNOWLEDGMENT}

This publication has emanated from research supported in part by a research grant from Science Foundation Ireland (SFI) and is co-funded under the European Regional Development Fund under Grant Number 13/RC/2077.

\section{REFERENCES}

[1] M. Shanmukhi and O. B. V. Ramanaiah, "A Survey on Energy Efficient Data Aggregation Protocols for Wireless Sensor Networks", International Journal of Applied Engineering Research, 11(10), Research India Publications, 2016, pp. 6990-7002.

[2] R. Rajagopalan and P.K. Varshney, "Data aggregation techniques in sensor networks: A survey", Electrical Engineering and Computer Science, Paper 22, Syracuse University, 2006.

[3] M.Dagar and S. Mahajan, "Data Aggregation in Wireless Sensor Network: A Survey", International Journal of Information and Computation Technology, 3(3), International Research Publications House, 2013, pp. 167-174.

[4] G. Dhand and S.S. Tyagi, "Data aggregation techniques in WSN:Survey", in Proc. 2nd International Conference on Intelligent Computing, Communication \& Convergence, ELSEVIER, 2016, pp. 378-384.
[5] E. Fasolo, et al., "IN-NETWORK AGGREGATION TECHNIQUES FOR WIRELESS SENSOR NETWORKS: A SURVEY", IEEE Wireless Communications, April, IEEE, 2007, pp. 70-87.

[6] S. Madden et al., "TAG: a Tiny AGgregation Service for Ad Hoc Sensor Networks", in Proc. of the 5th symposium on Operating systems design and implementation, ACM, 2002, pp. 131-146.

[7] B. Sun et al., "Data Aggregation Scheme Based on Compressive Sensing in Wireless Sensor Network", in Proc. 9th International Symposium on Computational Intelligence and Design, IEEE, 2016, pp. 453-456.

[8] N. Chitradevi et al., "Outlier aware data aggregation in distributed wireless sensor network using robust principal component analysis", in Proc. 2010 Second International conference on Computing, Communication and Networking Technologies, IEEE, 2010, pp. 1-9.

[9] Y. Li, Z. Yang and Q. Zhang, "Balenced Data Aggregation Method for WSN Based on Compressed Network Coding", in Proc. 2016 IEEE Advanced Information Management, Communicates, Electronic and Automation Control Conference (IMCEC), IEEE, 2016, pp.940-944.

[10] S. Brown and C.J. Sreenan, "A Study on Data Aggregation and Reliability in Managing Wireless Sensor Networks", in Proc. 2007 IEEE International Conference on Mobile Adhoc and Sensor Systems, IEEE, 2007, pp. 1-7.

[11] C. Florens and R. McEliece, "Packets distribution algorithms for sensor networks", in Proc. 22nd Annual Joint Conf. of the IEEE Computer and Communications Societies, vol. 2, IEEE, 2003, pp. 1063-1072.

[12] Y. Zhu, K. Sundaresan, and R. Sivakumar, "Practical Limits on Achievable Energy Improvements and Useable Delay Tolerance in Correlation Aware Data Gathering in Wireless Sensor Networks", in Proc. IEEE SECON 2005, IEEE, 2005, pp. 328-339.

[13] W. B. Heinzelman, A. P. Chandrakasan, and H. Balakrishnan, "An application-Specific Protocol Architecture for Wireless Microsensor Networks", IEEE Trans. Wireless Commun., 1(4), IEEE, 2002, pp. 660-70.

[14] S. Nath et al., "Synopsis Diffusion for Robust Aggregation in Sensor Networks", in Proc. ACM SenSys 2004, ACM, 2004, pp. 250-262.

[15] H. Rahman, N. Ahmed, and Dd. I. Hussain, "A Hybrid Data Aggregation Scheme for Internet of Things (IoT)", in Proc. 2016 IEEE Annual India Conference (INDICON), IEEE, 2016, pp. 1-6.

[16] N. Vidhya and Dr. P. Sengottuvelan, "A Survey on Secure Data Aggregation Techniques in Wireless Sensor Networks", International Journal of Innovative Research in Computer and Communication Engineering, 3(10), IJIRCCE, pp.9171-9181.

[17] J. Jose, J. Jose and F. Jose, "A Survey on Secure Data Aggregation Protocols in Wireless Sensor Networks", International Journal of Computer Applications, 55(18), IJCA, 2012.

[18] R. K. Ranjan and S. P. Karmore, "Survey on secured data aggregation in wireless sensor network", in Proc. 2015 International Conference on Innovations in Information, Embedded and Communication Systems (ICIIECS), IEEE, 2015, pp.1-4.

[19] C-Y. Wan, A.T. Campbell, L. Krishnamurthy,’PSFQ: A Reliable Transport Protocol for Wireless Sensor Networks", in Proc. of the 1st ACM international workshop on Wireless sensor networks and applications, ACM, 2002, pp. 862-872

[20] K.M. Azharul Hasan, J. Rabbi and Sk. Md. Masudul Ahsan, "Incremental aggregation scheme based on Extendible Karnaugh Arrays", in Proc. 17th International Conference on Computer and Information Technology, IEEE, 2014, pp. 105-109

[21] J.M. Bahi, A. Makhoul and M. Medlej, "Data aggregation for periodic sensor networks using sets similarity functions", in Proc. 7th International Wireless Communications and Mobile Computing Conference, IEEE, 2011, pp. 559-564

[22] I.Atoui et al, "Tree-based data aggregation approach in wireless sensor network using fitting functions", in Proc. of the Sixth International Conference on Digital Information Processing and Communications, IEEE, 2016 pp. 146-150

[23] M. Schwartz, Telecommunication Networks: Protocols, Modeling and Analysis, Addison-Wesley, 1987.

[24] IEEE Standard for Low Rate Wireless Networks, IEEE Std. 802.15.42015, IEEE, 2016

[25] K. Guo, P. Zhang and J. Ma, "Secure and Cost-Effective Distributed Aggregation for Mobile Sensor Networks", Sensors, 16(4), MDPI, 2016, pp. $1-27$.

[26] D. E. Knuth, The Art of Computer Programming, Vol. 2: Seminumerical Algorithms, 3rd Edn, Addison-Wesley, 1998, p. 232. 